\title{
ON THE UNIFORM CONVERGENCE OF FOURIER SERIES EXPANSIONS IN THE SYSTEM OF EIGENFUNCTIONS OF THE EQUATION OF A VIBRATING ROD AT ONE END OF WHICH THE MASS IS CONCENTRATED
}

\author{
KONUL F. ABDULLAYEVA, ZIYATKHAN S. ALIYEV, AND NAZIM B. KERIMOV
}

\begin{abstract}
In this paper we consider a spectral problem for ordinary differential equations of fourth order with a spectral parameter in the boundary condition. This problem arises when variables are separated in the dynamical boundary value problem describing bending vibrations of a homogeneous rod, in cross-sections of which the longitudinal force acts, the left end of which is fixed and on the right end a mass is concentrated. We study the uniform convergence of the spectral expansions in terms of eigenfunctions of this problem.
\end{abstract}

\section{Introduction}

Consider the homogeneous Euler-Bernoulli beam of length $L$, density $\rho$ and cross-sectional area $F$, the left end of which is fixed rigidly, and on the right end of which load with mass of $m$ is concentrated.

The free bending vibrations of a homogeneous rod of constant rigidity, in cross sections of which the longitudinal force acts, is described by the equation $[11, \mathrm{Ch}$. $8, \S 5$, formula $(84)]$

$$
E J \frac{\partial^{4} U(X, t)}{\partial X^{4}}-\frac{\partial}{\partial X}\left(\tilde{Q}(X) \frac{\partial U(X, t)}{\partial X}\right)+\rho F \frac{\partial^{2} U(X, t)}{\partial t^{2}}=0,
$$

where $U(X, t)$ is a flexure of the current point of axis of the rod, $E J$ is the flexural rigidity of the $\operatorname{rod}, \tilde{Q}(X)$ is longitudinal force, $\rho$ is rod density, $F$ is a cross-sectional area.

If the left end is fixed rigidly and at the right end is concentrated a load with mass of $m$, then the boundary conditions can be written in the following form $[11$, Ch. $8, \S 5$, Table 6, p. 154]

$$
U(0, t)=0, \frac{\partial U(0, t)}{\partial X}=0,
$$

2010 Mathematics Subject Classification. 34B05, 34B08, 34B24, 34L10, 34L20 .

Key words and phrases. ordinary differential equations, the bending vibrations of a homogeneous rod, eigenfunction, Fourier series, uniform convergence. 


$$
E J \frac{\partial^{2} U(L, t)}{\partial X^{2}}=0, E J \frac{\partial^{3} U(L, t)}{\partial X^{3}}-\tilde{Q}(L) \frac{\partial U(L, t)}{\partial X}=-m \frac{\partial^{2} U(L, t)}{\partial t^{2}} .
$$

Introducing the notation $x=\frac{X}{L}, u=\frac{U}{L}$ we write the equation of free bending vibrations of a homogeneous rod and the boundary conditions in the form

$$
\begin{gathered}
\frac{\partial^{4} u(x, t)}{\partial x^{4}}-\frac{\partial}{\partial x}\left(Q(x) \frac{\partial u(x, t)}{\partial x}\right)+\frac{\rho F L^{4}}{E J} \frac{\partial^{2} u(x, t)}{\partial t^{2}}=0, \\
u(0, t)=0, \frac{\partial u(0, t)}{\partial x}=0, \frac{\partial^{2} u(1, t)}{\partial x^{2}}=0, \\
\frac{\partial^{3} u(1, t)}{\partial x^{3}}-Q(1) \frac{\partial u(1, t)}{\partial x}=-\frac{m L^{3}}{E J} \frac{\partial^{2} u(1, t)}{\partial t^{2}},
\end{gathered}
$$

where $Q(x)=\frac{L^{2}}{E J} \tilde{Q}(L x)$.

Let $\lambda=\rho F L^{4} \omega^{2} / E J$. Then this problem with substitution $u(x, t)=y(x) \cos \omega t$ is reduced (see, e.g., [11, Ch. 11, $\S 2$, formula (12)]) to the following eigenvalue problem

$$
\begin{gathered}
y^{(4)}(x)-\left(q(x) y^{\prime}(x)\right)^{\prime}=\lambda y(x), 0<x<1, \\
U_{1}(y) \equiv y(0)=0, U_{2}(y) \equiv y^{\prime}(0)=0, \\
U_{3}(y) \equiv y^{\prime \prime}(1)=0, \\
U_{4}(y) \equiv T y(1)-a \lambda y(1)=0,
\end{gathered}
$$

where $q(x) \equiv Q(x), T y \equiv y^{\prime \prime \prime}-q y^{\prime}, a=\frac{m}{\rho F L}$.

It is obvious that the following conditions are satisfied

$$
q(x)>0, x \in[0,1], a>0 .
$$

Moreover, we suppose that the function $q(x)$ is absolutely continuous on $[0,1]$.

The location of eigenvalues in the complex plane (on the real axis), the structure of the root subspaces, the asymptotic formulas for eigenvalues and eigenfunctions, the basis property of subsystems of eigenfunctions of problem (1.1)-(1.4) in more general form were investigated in the paper [1]. In [1], in particular, a necessary and sufficient condition is established for the system of eigenfunctions of this problem after removing one function to form a basis in $L_{p}(0,1), 1<p<\infty$.

One of the most common methods for solving partial differential equations is the Fourier method. The justification of this method in boundary value problems for partial differential equations is based on the proof of the convergence of spectral expansions in the subsystems of root functions of the corresponding eigenvalue problems in various function spaces. The subject of the present paper is the study of uniform convergence of Fourier series expansions for continuous functions in the subsystems of eigenfunctions of problem (1.1)-(1.4).

The spectral properties of ordinary differential operators with spectral parameter in the boundary conditions were studied by many authors (see, e.g., [1-8, $12,13,15-20,22-25,30,31,33-36])$. The basis properties of subsystems of root functions in the space $L_{p}(0,1), 1<p<\infty$, of Sturm-Liouville problems with a spectral parameter in the boundary conditions were studied in $[2,3,13,15-20,25$, $30]$, of ordinary differential operators of fourth order with spectral parameter in the boundary conditions were studied in $[1,4,5,7,22]$. The uniform convergence of Fourier expansions in terms of root functions of Sturm-Liouville operators with a spectral parameter in the boundary conditions were investigated in $[13,16-18$, 
$20,23,24,30]$, and of ordinary differential operators of fourth order when the spectral parameter is not contained in the boundary conditions in [28, 29] (see also their references). The uniform convergence of Fourier series expansions of continuous functions in the subsystems of eigenfunctions of differential operators of fourth order with a spectral parameter in the boundary conditions have been investigated in recent papers $[6,33]$.

In this paper, it is shown that the uniform convergence of spectral expansions in terms of eigenfunctions of problem (1.1)-(1.4) reduces to studying the uniform convergence of Fourier series in the functions of one particular system.

The structure of this paper is as follows. In Section 2 we give the spectral properties (the location of eigenvalues on real axis, the structure of root subspaces, the asymptotic formulas for eigenvalues and eigenfunctions, the oscillation properties of eigenfunctions and the basis properties of eigenfunctions in $\left.L_{p}(0,1), 1<p<\infty\right)$ of problem (1.1)-(1.4) and problem (1.1)-(1.3) with the addition of the "asymptotic" boundary condition $y(1)=0$ which have been studied in $[1,10,22]$. It should be noted that in order to study the uniform convergence of spectral expansions in eigenfunctions of problem (1.1)-(1.4) are not beneficial to us the asymptotic formulas for the eigenvalues and eigenfunctions of problems (1.1)-(1.4) and (1.1)-(1.3), $y(1)=0$, which were obtained earlier in [1]. In Sections 3 and 4 we obtain refined asymptotic formulas for the eigenvalues and eigenfunctions of problems (1.1)-(1.3) (with $q(x) \equiv 0), y(1)=0$, and (1.1)-(1.4) respectively. In Section 5 we find a sufficient condition and also necessary and sufficient condition for the uniform convergence of Fourier series expansions for continuous functions in the subsystems of eigenfunctions of problem (1.1)-(1.4). Here we also establish a necessary and sufficient condition for the uniform convergence of Fourier series expansions of a continuous function in the systems of functions which is adjoint to the subsystems of eigenfunctions of problem (1.1)(1.4).

\section{Some auxiliary facts and statements}

We consider the boundary condition

$$
\tilde{U}_{4}(y) \equiv y(1)=0 .
$$

Alongside the problem (1.1)-(1.4) we shall consider the problem (1.1)-(1.3), (2.1). Problem (1.1)-(1.3), (2.1) has been considered before in [9, 10, 14], where the authors study the oscillation properties of the eigenfunctions and their derivatives.

The following theorems are special cases of the general results of [10] and [1]. Theorem 2.1. (see [10, Theorem 5.4 and 5.5]) The eigenvalues of the problem (1.1)-(1.3), (2.1) are real, simple and form an infinitely increasing sequence $\left\{\eta_{k}\right\}_{k=1}^{\infty}$ such that $\eta_{k}>0$ for $k \in \mathbb{N}$. Moreover, the eigenfunction $v_{k}(x), k \in \mathbb{N}$, corresponding to the eigenvalue $\eta_{k}$ has precisely $k-1$ simple zeros in the interval $(0,1)$.

Theorem 2.2. (see [1, Theorem 4.1] and [22, Theorem 3.1]) The eigenvalues of the problem (1.1)-(1.4) are real, simple and form an infinitely increasing sequence 
$\left\{\lambda_{k}\right\}_{k=1}^{\infty}$ such that $\lambda_{1}<0$ and $\lambda_{k}>0$ for $k \geq 2$. Moreover, the eigenfunction $y_{k}(x), k \geq 2$, corresponding to the eigenvalue $\lambda_{k}$ has precisely $k-1$ simple zeros in the interval $(0,1)$.

Theorem 2.3. (see [1, Theorem 5.1]) The following asymptotic formulas hold:

$$
\begin{gathered}
\sqrt[4]{\eta_{k}}=\left(k+\frac{1}{4}\right) \pi+O\left(\frac{1}{k}\right), \\
\sqrt[4]{\lambda_{k}}=\left(k-\frac{3}{4}\right) \pi+O\left(\frac{1}{k}\right), \\
v_{k}(x)=\sin \left(k+\frac{1}{4}\right) \pi x-\cos \left(k+\frac{1}{4}\right) \pi x+e^{-\left(k+\frac{1}{4}\right) \pi x}+O\left(\frac{1}{k}\right), \\
y_{k}(x)=\sin \left(k-\frac{3}{4}\right) \pi x-\cos \left(k-\frac{3}{4}\right) \pi x+e^{-\left(k-\frac{3}{4}\right) \pi x}+O\left(\frac{1}{k}\right),
\end{gathered}
$$

where relations (2.4)-(2.5) hold uniformly for $x \in[0,1]$.

Theorem 2.4. (see [1, Lemmas 6.1-6.3, Corollary 6.1, Theorem 6.2]) Let $r$ be an arbitrarily fixed natural number. Then the system of eigenfunctions $\left\{y_{k}(x)\right\}_{k=1, k \neq r}^{\infty}$ of problem (1.1)-(1.4) forms a basis in the space $L_{p}(0,1), 1<p<\infty$, which is an unconditional basis for $p=2$.

Remark 2.1. It should be noted that, using the asymptotic formulas (2.4) and (2.5), it is impossible to find sufficient conditions for the uniform convergence of Fourier series expansions of continuous functions in the system of eigenfunctions of problem (1.1)-(1.4) after removing one function. Therefore, in order to establish these conditions we need to obtain the asymptotic formulas for the eigenpairs $\left(\eta_{k}, v_{k}(x)\right)$ and $\left(\lambda_{k}, y_{k}(x)\right)$ of problems (1.1)-(1.3), (2.1) and (1.1)-(1.4) respectively up to the term with $\frac{1}{k^{2}}$.

\section{Refinement of asymptotic formulas for eigenvalues and eigenfunctions of the boundary-value problem (1.1)-(1.3), (2.1)$$
\text { with } q(x) \equiv 0
$$

In this section we consider the spectral problem (1.1)-(1.3), (2.1) with $q(x) \equiv 0$. Lemma 3.1 For sufficiently large $k \in \mathbb{N}$ for the eigenvalues $\eta_{k}$ and corresponding eigenfunctions $v_{k}(x)$ of problem (1.1)-(1.3), (2.1) with $q(x) \equiv 0$ the following asymptotic formulas hold:

$$
\begin{gathered}
\sqrt[4]{\eta_{k}}=\left(k+\frac{1}{4}\right) \pi+O\left(e^{-k \pi}\right) \\
v_{k}(x)=\sin \left(k+\frac{1}{4}\right) \pi x-\cos \left(k+\frac{1}{4}\right) \pi x+e^{-\left(k+\frac{1}{4}\right) \pi x}+O\left(e^{-k \pi}\right),
\end{gathered}
$$

where relation (3.2) holds uniformly for $x \in[0,1]$.

Proof. In the case $q \equiv 0$ equation (1.1) has four linearly independent solutions

$$
z_{k}(x, \rho)=e^{\rho \omega_{k} x}, k=1,2,3,4,
$$

where $\rho=\sqrt[4]{\lambda}$ and $\omega_{k}, k=1,2,3,4$, are distinct fourth roots of unity:

$$
\omega_{1}=-\omega_{4}=-1, \omega_{2}=-\omega_{3}=-i \text {. }
$$


Hence we have

$$
z_{k}^{(s)}(x, \rho)=e^{\rho \omega_{k} x}\left(\rho \omega_{k}\right)^{s}, k=1,2,3,4, s=0,1,2,3 .
$$

Then by (3.4) it follows from (1.2), (1.3) and (2.1) that

$$
\begin{aligned}
& U_{1}\left(z_{k}\right) \equiv z_{k}(0, \rho)=1, U_{2}\left(z_{k}\right) \equiv z_{k}^{\prime}(0, \rho)=\rho \omega_{k}, \\
& U_{3}\left(z_{k}\right) \equiv z_{k}^{\prime \prime}(1)=\rho^{2} \omega_{k}^{2} e^{\rho \omega_{k}}, \tilde{U}_{4}\left(z_{k}\right) \equiv z_{k}(1)=e^{\rho \omega_{k}} .
\end{aligned}
$$

Now we consider the characteristic determinant

$$
\Delta(\lambda)=\left|\begin{array}{cccc}
U_{1}\left(z_{1}\right) & U_{1}\left(z_{2}\right) & U_{1}\left(z_{3}\right) & U_{1}\left(z_{4}\right) \\
U_{2}\left(z_{1}\right) & U_{2}\left(z_{2}\right) & U_{2}\left(z_{3}\right) & U_{2}\left(z_{4}\right) \\
U_{3}\left(z_{1}\right) & U_{3}\left(z_{2}\right) & U_{3}\left(z_{3}\right) & U_{3}\left(z_{4}\right) \\
\tilde{U}_{4}\left(z_{1}\right) & \tilde{U}_{4}\left(z_{2}\right) & \tilde{U}_{4}\left(z_{3}\right) & \tilde{U}_{4}\left(z_{4}\right)
\end{array}\right|
$$

By (3.3) and (3.5), from (3.6) we obtain

$$
\begin{gathered}
\Delta(\lambda)=\rho^{3}\left|\begin{array}{cccc}
1 & 1 & 1 & 1 \\
-1 & -i & i & 1 \\
e^{-\rho} & -e^{-i \rho} & -e^{i \rho} & e^{\rho} \\
e^{-\rho} & e^{-i \rho} & e^{i \rho} & e^{\rho}
\end{array}\right|= \\
2 \rho^{3} e^{\rho}\left\{(1+i) e^{-i \rho}-(1-i) e^{i \rho}+O\left(e^{-\rho}\right)\right\} .
\end{gathered}
$$

Thus, the roots of the characteristic determinant $\Delta(\lambda)$ are the roots of the following equation

$$
(1+i) e^{-i \rho}-(1-i) e^{i \rho}+O\left(e^{-\rho}\right)=0 .
$$

It is easy to show that this equation is equivalent to the following equation

$$
e^{2 i \rho}=i+O\left(e^{-\rho}\right) \text {. }
$$

By virtue of (2.2) we have

$$
\rho_{k}=\sqrt[4]{\eta_{k}}=\left(k+\frac{1}{4}\right) \pi+\varepsilon_{k}
$$

where $\varepsilon_{k}=o(1)$.

Since $\rho_{k}, k \in \mathbb{N}$, are the roots of $\Delta(\lambda)$, taking (3.8) into account, from (3.7) we obtain

which implies that

$$
e^{2 i \varepsilon_{k}}=1+O\left(e^{-k \pi}\right)
$$

$$
\varepsilon_{k}=O\left(e^{-k \pi}\right) .
$$

Then from (3.8) and (3.9) we obtain the asymptotic formula (3.1).

By virtue of (3.1) we have the following relations

$$
\begin{aligned}
& e^{i \rho_{k}}=\frac{\sqrt{2}}{2}(-1)^{k}(1+i)+O\left(e^{-k \pi}\right), \\
& e^{-i \rho_{k}}=\frac{\sqrt{2}}{2}(-1)^{k}(1-i)+O\left(e^{-k \pi}\right) .
\end{aligned}
$$


Now we show that the asymptotic formula (3.2) is true. It is known that the eigenfunction $v(x, \rho)$ corresponding to the eigenvalue $\lambda=\rho^{4}$ has the form

$$
v(x, \rho)=C_{\rho}\left|\begin{array}{cccc}
z_{1}(x, \rho) & z_{2}(x, \rho) & z_{3}(x, \rho) & z_{4}(x, \rho) \\
U_{1}\left(z_{1}\right) & U_{1}\left(z_{2}\right) & U_{1}\left(y_{3}\right) & U_{1}\left(z_{4}\right) \\
U_{2}\left(z_{1}\right) & U_{2}\left(z_{2}\right) & U_{2}\left(z_{3}\right) & U_{2}\left(z_{4}\right) \\
\tilde{U}_{4}\left(z_{1}\right) & \tilde{U}_{4}\left(z_{2}\right) & \tilde{U}_{4}\left(z_{3}\right) & \tilde{U}_{4}\left(z_{4}\right)
\end{array}\right|,
$$

where $C_{\rho}$ is an arbitrary nonzero constant that depends on $\rho$.

By virtue of (3.1), (3.3)-(3.5) and (3.10) it follows from (3.11) that

$$
\begin{gathered}
v_{k}(x)=v\left(x, \rho_{k}\right)=C_{\rho_{k}} \rho_{k} e^{\rho_{k}}\left\{\left|\begin{array}{cccc}
e^{-\rho_{k} x} & e^{-i \rho_{k} x} & e^{i \rho_{k} x} & e^{\rho_{k}(x-1)} \\
1 & 1 & 1 & 0 \\
-1 & -i & i & 0 \\
0 & e^{-i \rho_{k}} & e^{i \rho_{k}} & 1
\end{array}\right|+O\left(e^{-\rho_{k}}\right)\right\}= \\
2 i C_{\rho_{k}} \rho_{k} e^{\rho_{k}}\left\{\sin \left(k+\frac{1}{4}\right) \pi x-\cos \left(k+\frac{1}{4}\right) \pi x+e^{-\left(k+\frac{1}{4}\right) \pi x}+O\left(e^{-k \pi}\right)\right\} .
\end{gathered}
$$

Considering (2.4) we can choose the constant $C_{\rho_{k}}$ as follows: $C_{\rho_{k}}=-\frac{-i e^{-\rho_{k}}}{2 \rho_{k}}$. Then from the last relation we obtain the asymptotic formula (3.2). The proof of this lemma is complete.

In view of $(3.2)$ we get

$$
\left\|v_{k}(x)\right\|_{2}^{2}=\int_{0}^{1} v_{k}^{2}(x) d x=1+O\left(e^{-k \pi}\right) .
$$

Remark 3.1. Let

$$
\Phi_{k}(x)=v_{k}(x)\left\|v_{k}(x)\right\|_{2}^{-1}, k \in \mathbb{N},
$$

i.e. $\Phi_{k}(x), k \in \mathbb{N}$, is a normalized eigenfunction corresponding to the eigenvalue $\eta_{k}$ of problem (1.1)-(1.3), (2.1) with $q \equiv 0$. Then it follows from (3.2) and (3.12) that

$$
\Phi_{k}(x)=\sin \left(k+\frac{1}{4}\right) \pi x-\cos \left(k+\frac{1}{4}\right) \pi x+e^{-\left(k+\frac{1}{4}\right) \pi x}+O\left(e^{-k \pi}\right) .
$$

\section{Refinement of asymptotic formulas for eigenvalues and eigenfunctions of the boundary-value problem (1.1)-(1.4)}

In this section we consider the eigenvalue problem (1.1)-(1.4).

Lemma 4.1 For sufficiently large $k \in \mathbb{N}$ for the eigenvalues $\lambda_{k}$ and corresponding eigenfunctions $y_{k}(x)$ of problem (1.1)-(1.4) the following asymptotic formulas hold:

$$
\sqrt[4]{\lambda_{k}}=\left(k-\frac{3}{4}\right) \pi+\frac{q_{0}-2 / a}{4 k \pi}+O\left(\frac{1}{k^{2}}\right)
$$




$$
\begin{gathered}
y_{k}(x)=\sin \left(k-\frac{3}{4}\right) \pi x-\cos \left(k-\frac{3}{4}\right) \pi x+e^{-\left(k-\frac{3}{4}\right) \pi x}+ \\
\frac{\left(q_{0}-2 / a\right) x-q_{0}(x)}{4 k \pi} \sin \left(k-\frac{3}{4}\right) \pi x+\frac{\left(q_{0}-2 / a\right) x-q_{0}(x)}{4 k \pi} \cos \left(k-\frac{3}{4}\right) \pi x- \\
\frac{\left(q_{0}-2 / a\right) x+q_{0}(x)}{4 k \pi} e^{-\left(k-\frac{3}{4}\right) \pi x}+O\left(\frac{1}{k^{2}}\right),
\end{gathered}
$$

where $q_{0}(x)=\int_{0}^{x} q(t) d t, q_{0}=\int_{0}^{1} q(x) d x$, and relation (4.2) holds uniformly for $x \in[0,1]$.

Proof. Since all eigenvalues of problem (1.1)-(1.4), except for the first eigenvalue, are positive, in equation (1.1) we set $\lambda=\mu^{4}$ with $\mu>0$. As is known (see [32, Ch. II, $\S 4.5$ Theorem 1 and $\S 4.6$ formulas (27)-(29)]), equation (1.1) has four linearly independent solutions $\varphi_{k}(x, \mu), k=1,2,3,4$, regular in $\mu$ (for sufficiently large $\mu>0)$ and satisfying the relations

$$
\begin{gathered}
\varphi_{k}^{(s)}(x, \mu)=\left(\mu \omega_{k}\right)^{s} e^{\mu \omega_{k} x}\left\{1+\frac{q_{0}(x)}{4 \mu \omega_{k}}+O\left(\frac{1}{\mu^{2}}\right)\right\} \\
k=1,2,3,4, s=0,1,2,3
\end{gathered}
$$

where $\omega_{1}=-\omega_{4}=-1, \omega_{2}=-\omega_{3}=-i(\operatorname{see}(3.4))$.

By virtue of (4.3) and boundary conditions (1.2)-(1.4) we have

$$
\begin{aligned}
& U_{1}\left(\varphi_{k}\right)=1+O\left(\mu^{-2}\right), U_{2}\left(\varphi_{k}\right)=\mu \omega_{k}\left(1+O\left(\mu^{-2}\right)\right), \\
& U_{3}\left(\varphi_{k}\right)=\mu^{2} \omega_{k}^{2} e^{\rho \omega_{k}}\left(1+\frac{q_{0}}{4 \mu \omega_{k}}+O\left(\mu^{-2}\right)\right) \\
& U_{4}\left(\varphi_{k}\right)=-a \mu^{4} e^{\mu \omega_{k}}\left(1+\frac{q_{0}-4 / a}{4 \mu \omega_{k}}+O\left(\mu^{-2}\right)\right) .
\end{aligned}
$$

Taking (4.3) and (4.4) into account for the characteristic determinant

$$
\Delta(\lambda)=\left|\begin{array}{cccc}
U_{1}\left(\varphi_{1}\right) & U_{1}\left(\varphi_{2}\right) & U_{1}\left(\varphi_{3}\right) & U_{1}\left(\varphi_{4}\right) \\
U_{2}\left(\varphi_{1}\right) & U_{2}\left(\varphi_{2}\right) & U_{2}\left(\varphi_{3}\right) & U_{2}\left(\varphi_{4}\right) \\
U_{3}\left(\varphi_{1}\right) & U_{3}\left(\varphi_{2}\right) & U_{3}\left(\varphi_{3}\right) & U_{3}\left(\varphi_{4}\right) \\
U_{4}\left(\varphi_{1}\right) & U_{4}\left(\varphi_{2}\right) & U_{4}\left(\varphi_{3}\right) & U_{4}\left(\varphi_{4}\right)
\end{array}\right|
$$

we have the following relation

$$
\begin{aligned}
& \Delta(\lambda)=a \mu^{7} e^{\mu} \times \\
& \left\{\begin{array}{cccc}
1 & 1 & 1 & 0 \\
-1 & -i & i & 0 \\
0 & -e^{-i \mu}\left(1-\frac{q_{0}}{4 \mu i}\right) & -e^{i \mu}\left(1+\frac{q_{0}}{4 \mu i}\right) & 1+\frac{q_{0}}{4 \mu} \\
0 & e^{-i \mu}\left(1-\frac{q_{0}-4 / a}{4 \mu i}\right) & e^{i \mu}\left(1+\frac{q_{0}-4 / a}{4 \mu i}\right) & 1+\frac{q_{0}-4 / a}{4 \mu}
\end{array} \mid+O\left(\mu^{-2}\right)\right\}= \\
& -a \mu^{7} e^{\mu}\left\{\left|\begin{array}{ccc}
1-i & 1+i & 0 \\
-e^{-i \mu}\left(1-\frac{q_{0}}{4 \mu i}\right) & -e^{i \mu}\left(1+\frac{q_{0}}{4 \mu i}\right) & 1+\frac{q_{0}}{4 \mu} \\
e^{-i \mu}\left(1-\frac{q_{0}-4 / a}{4 \mu i}\right) & e^{i \mu}\left(1+\frac{q_{0}-4 / a}{4 \mu i}\right) & 1+\frac{q_{0}-4 / a}{4 \mu}
\end{array}\right|+O\left(\mu^{-2}\right)\right\}=
\end{aligned}
$$




$$
\begin{gathered}
2 a \mu^{7} e^{\mu}\left\{(1-i) e^{i \mu}\left\{1+\frac{q_{0}-2 / a}{4 \mu i}(1+i)\right\}-\right. \\
\left.(1+i) e^{-i \mu}\left\{1-\frac{q_{0}-2 / a}{4 \mu i}(1-i)\right\}+O\left(\mu^{-2}\right)\right\} .
\end{gathered}
$$

Then it follows that the roots of the characteristic determinant $\Delta(\lambda)$ are the roots of the equation

$$
\begin{gathered}
(1-i) e^{i \mu}\left\{1+\frac{q_{0}-2 / a}{4 \mu i}(1+i)\right\}- \\
(1+i) e^{-i \mu}\left\{1-\frac{q_{0}-2 / a}{4 \mu i}(1-i)\right\}+O\left(\mu^{-2}\right)=0 .
\end{gathered}
$$

This equation is equivalent to the following equation

$$
e^{2 i \mu}=i-\frac{q_{0}-2 / a}{2 \mu}+O\left(\mu^{-2}\right) .
$$

In view of (2.3) we have

$$
\mu_{k}=\sqrt[4]{\lambda_{k}}=\left(k-\frac{3}{4}\right) \pi+\varepsilon_{k}
$$

where $\varepsilon_{k}=o(1)$. By (4.7) from (4.6) we obtain

$$
e^{2 i \varepsilon_{k}}=1-\frac{q_{0}-2 / a}{2 k \pi i}+O\left(\frac{1}{k^{2}}\right)
$$

which implies that

$$
\varepsilon_{k}=\frac{q_{0}-2 / a}{4 k \pi}+O\left(\frac{1}{k^{2}}\right) .
$$

Thus, formula (4.1) follows from relations (4.7) and (4.8).

It follows from (4.1) that

$$
\begin{aligned}
& e^{-i \mu_{k}}=-\frac{\sqrt{2}}{2}(-1)^{k}(1-i)+O\left(e^{-k \pi}\right), \\
& e^{i \mu_{k}}=-\frac{\sqrt{2}}{2}(-1)^{k}(1+i)+O\left(e^{-k \pi}\right) .
\end{aligned}
$$

Now we prove the asymptotic formula (4.2). It is obvious that the eigenfunction $y(x, \mu)$ corresponding to the eigenvalue $\lambda=\mu^{4}$ has the form

$$
y(x, \mu)=D_{\mu}\left|\begin{array}{llll}
\varphi_{1}(x, \mu) & \varphi_{2}(x, \mu) & \varphi_{3}(x, \mu) & \varphi_{4}(x, \mu) \\
U_{1}\left(\varphi_{1}\right) & U_{1}\left(\varphi_{2}\right) & U_{1}\left(\varphi_{3}\right) & U_{1}\left(\varphi_{4}\right) \\
U_{2}\left(\varphi_{1}\right) & U_{2}\left(\varphi_{2}\right) & U_{2}\left(\varphi_{3}\right) & U_{2}\left(\varphi_{4}\right) \\
U_{4}\left(\varphi_{1}\right) & U_{4}\left(\varphi_{2}\right) & U_{4}\left(\varphi_{3}\right) & U_{4}\left(\varphi_{4}\right)
\end{array}\right| .
$$

where $D_{\mu}$ is an arbitrary nonzero constant that depends on $\mu$.

By (4.1), (4.3) and (4.9) from (4.10) we obtain

$$
\begin{gathered}
y\left(x, \mu_{k}\right)=a \mu_{k}^{5} e^{\mu_{k}} D_{\mu_{k}} \times \\
\left\{\left|\begin{array}{cccc}
e^{-\mu_{k} x}\left(1-\frac{q_{0}(x)}{4 k \pi}\right) & e^{-i \mu_{k} x}\left(1-\frac{q_{0}(x)}{4 k \pi i}\right) & e^{i \mu_{k} x}\left(1+\frac{q_{0}(x)}{4 k \pi i}\right) & e^{\mu_{k}(x-1)} \\
1 & 1 & 1 & 0 \\
0 & 1-i & 1+i & 0 \\
0 & \frac{\sqrt{2}}{2}(-1)^{k}(1-i) & \frac{\sqrt{2}(x)}{2}(-1)^{k}(1+i) & 1+\frac{q_{0}-4 / a}{4 k \pi}
\end{array}\right|+\right.
\end{gathered}
$$




$$
\begin{gathered}
\left.O\left(\frac{1}{k^{2}}\right)\right\}=2 i a \mu_{k}^{5} e^{\mu_{k}} D_{\mu_{k}}\left(1+\frac{q_{0}}{4 k \pi}\right) \times \\
\left\{\left(1-\frac{q_{0}(x)}{4 k \pi}\right) \sin \mu_{k} x-\left(1+\frac{q_{0}(x)}{4 k \pi}\right) \cos \mu_{k} x+\right. \\
\left.\left(1-\frac{q_{0}(x)}{4 k \pi}\right) e^{-\mu_{k} x}+O\left(\frac{1}{k^{2}}\right)\right\}
\end{gathered}
$$

Considering relation $(2.5)$ we can choose $D_{\mu_{k}}$ to be $D_{\mu_{k}}=\frac{\mu_{k}^{-5} e^{-\mu_{k}}\left(1+\frac{q_{0}}{4 k \pi}\right)^{-1}}{2 i a}$. Then from the last relation it follows that

$$
\begin{gathered}
y_{k}(x)=y\left(x, \mu_{k}\right)=\left(1-\frac{q_{0}(x)}{4 k \pi}\right) \sin \mu_{k} x-\left(1+\frac{q_{0}(x)}{4 k \pi}\right) \cos \mu_{k} x+ \\
\left(1-\frac{q_{0}(x)}{4 k \pi}\right) e^{-\mu_{k} x}+O\left(\frac{1}{k^{2}}\right) .
\end{gathered}
$$

In view of (4.1) we have the following relations

$$
\begin{gathered}
\sin \mu_{k} x=\sin \left(k-\frac{3}{4}\right) \pi x+\frac{\left(q_{0}-2 / a\right) x}{4 k \pi} \cos \left(k-\frac{3}{4}\right) \pi x+O\left(\frac{1}{k^{2}}\right), \\
\cos \mu_{k} x=\cos \left(k-\frac{3}{4}\right) \pi x+\frac{\left(q_{0}-2 / a\right) x}{4 k \pi} \sin \left(k-\frac{3}{4}\right) \pi x+O\left(\frac{1}{k^{2}}\right), \\
e^{-\mu_{k} x}=e^{-\left(k-\frac{3}{4}\right) \pi x}\left\{1-\frac{\left(q_{0}-2 / a\right) x}{4 k \pi}\right\}+O\left(\frac{1}{k^{2}}\right) .
\end{gathered}
$$

In view of these relations from (4.11) we obtain an asymptotic formula (4.2). The proof of this theorem is complete.

\section{Uniform convergence Fourier series in the system of eigenfunctions of problem (1.1)-(1.4)}

By (3.13) it follows from (4.2) that for $k \geq 2$ the following equality

$$
\begin{gathered}
y_{k}(x)=\Phi_{k-1}(x)+\frac{\left(q_{0}-2 / a\right) x-q_{0}(x)}{4 k \pi}\left\{\sin \left(k-\frac{3}{4}\right) \pi x+\cos \left(k-\frac{3}{4}\right) \pi x\right\}- \\
\frac{\left(q_{0}-2 / a\right) x+q_{0}(x)}{4 k \pi} e^{-\left(k-\frac{3}{4}\right) \pi x}+O\left(\frac{1}{k^{2}}\right)
\end{gathered}
$$

holds. By (3.13) and (5.1) we get

$$
\Phi_{k}(1)=O\left(e^{-k \pi}\right), y_{k}(1)=\frac{\sqrt{2}(-1)^{k}}{2 a k \pi}+O\left(\frac{1}{k^{2}}\right), y_{k}^{2}(1)=O\left(\frac{1}{k^{2}}\right) .
$$

Moreover, it follows from (4.11) that

$$
\begin{array}{r}
y_{k}^{2}(x)=1+\frac{q_{0}(x)}{2 k \pi} \cos 2 \mu_{k} x+\left(1-\frac{q_{0}(x)}{2 k \pi}\right) e^{-2 \mu_{k} x}-\sin 2 \mu_{k} x+ \\
2\left(1-\frac{q_{0}(x)}{2 k \pi}\right) e^{-\mu_{k} x} \sin \mu_{k} x-2 e^{-\mu_{k} x} \cos \mu_{k} x+O\left(\frac{1}{k^{2}}\right) .
\end{array}
$$


Integrating this equality from 0 to 1 (using integration by parts) and taking the relations

$$
\begin{gathered}
\int_{0}^{1} q_{0}(x) \cos 2 \mu_{k} x d x=O\left(\frac{1}{k}\right) ; \int_{0}^{1} e^{-2 \mu_{k} x} d x=\frac{1}{2 k \pi}+O\left(\frac{1}{k^{2}}\right) \\
\int_{0}^{1} q_{0}(x) e^{-2 \mu_{k} x} d x=O\left(\frac{1}{k^{2}}\right) ; \int_{0}^{1} \sin 2 \mu_{k} x d x=\frac{1}{2 k \pi}+O\left(\frac{1}{k^{2}}\right) ; \\
\int_{0}^{1} e^{-\mu_{k} x} \sin \mu_{k} x d x=\frac{1}{2 k \pi}+O\left(\frac{1}{k^{2}}\right) ; \int_{0}^{1} q_{0}(x) e^{-\mu_{k} x} \sin \mu_{k} x d x=O\left(\frac{1}{k}\right) ; \\
\int_{0}^{1} e^{-\mu_{k} x} \cos \mu_{k} x d x=\frac{1}{2 k \pi}+O\left(\frac{1}{k^{2}}\right),
\end{gathered}
$$

into account we obtain

$$
\left\|y_{k}\right\|_{2}^{2}=1+O\left(\frac{1}{k^{2}}\right) .
$$

Let $r$ be an arbitrary fixed natural number. Then it follows from the proof of Theorem 6.2 of $[1$, p. 776$]$ that an element $u_{k}(x)$ of the system $\left\{u_{k}(x)\right\}_{k=1, k \neq r}^{\infty}$ adjoint to the system $\left\{y_{k}(x)\right\}_{k=1, k \neq r}^{\infty}$ is given by the relation

$$
u_{k}(x)=\left\{\left\|y_{k}\right\|_{2}^{2}-a y_{k}^{2}(1)\right\}^{-1}\left\{y_{k}(x)-\frac{y_{k}(1)}{y_{r}(1)} y_{r}(x)\right\} .
$$

In view of (5.2) and (5.3) from (5.4) we get

$$
u_{k}(x)=y_{k}(x)-\frac{y_{k}(1)}{y_{r}(1)} y_{r}(x)+O\left(\frac{1}{k^{2}}\right) .
$$

It follows from [1, Theorem 6.2] that any continuous function $f(x)$ can be expanded in the following Fourier series in the system $\left\{y_{k}(x)\right\}_{k=1, k \neq r}^{\infty}$ of eigenfunctions of problem (1.1)-(1.4) that converges in the space $L_{p}, 1<p<\infty$ :

$$
f(x)=\sum_{k=1, k \neq r}^{\infty}\left(f, u_{k}\right) y_{k}(x) .
$$

We study the uniform convergence of the series (5.6). The main result of this paper is the following theorem.

Theorem 5.1. $\quad$ Let $r$ be an arbitrarily fixed natural number, $f(x) \in C[0,1]$ and $f(x)$ has a uniformly convergent Fourier series expansion in the system $\left\{\Phi_{k}(x)\right\}_{k=1}^{\infty}$ on the interval $[0,1]$. If $\left(f, y_{r}\right) \neq 0$, then the Fourier series (5.6) of function $f(x)$ in the system $\left\{y_{k}(x)\right\}_{k=1, k \neq r}^{\infty}$ is uniformly convergent on every interval $[0, c], 0<c<1$. Moreover, the series (5.6) is uniformly convergent on the interval $[0,1]$ if and only if $\left(f, y_{r}\right)=0$.

Remark 5.1. It is clear from the asymptotic formula (3.13) that if $f(x) \in$ $W_{1}^{2}[0,1]$ and $f(0)=f(1)=0$, then the function $f(x)$ has a uniformly convergent Fourier series expansion in the system $\left\{\Phi_{k}(x)\right\}_{k=1}^{\infty}$ on the interval $[0,1]$. 
Proof of Theorem 5.1. Note that the series (5.6) is uniformly convergent on $[0,1]$ if and only if the series

$$
f_{1}(x)=\sum_{k=r+1}^{\infty}\left(f, u_{k}\right) y_{k}(x) .
$$

is uniformly convergent on $[0,1]$.

By virtue of (5.5) and (5.7) we have

$$
\begin{gathered}
f_{1}(x)=\sum_{k=r+1}^{\infty}\left(f, u_{k}\right) y_{k}(x)=\sum_{k=r+1}^{\infty}\left(f, y_{k}\right) y_{k}(x)- \\
\frac{\left(f, y_{r}\right)}{y_{r}(1)} \sum_{k=r+1}^{\infty} y_{k}(1) y_{k}(x)=\sum_{k=r+1}^{\infty}\left(f, y_{k}\right) y_{k}(x)- \\
\frac{\sqrt{2}}{2 a \pi} \frac{\left(f, y_{r}\right)}{y_{r}(1)} \sum_{k=r+1}^{\infty} \frac{(-1)^{k}}{k} y_{k}(x) .
\end{gathered}
$$

It follows from (5.1) that

$$
y_{k}(x)=\Phi_{k-1}(x)+O\left(\frac{1}{k}\right) .
$$

Then taking (5.9) into account from (5.8) we obtain

$$
\begin{gathered}
f_{1}(x)=\sum_{k=r+1}^{\infty}\left(f, u_{k}\right) y_{k}(x)=\sum_{k=r+1}^{\infty}\left(f, y_{k}\right) \Phi_{k-1}(x)+ \\
\sum_{k=r+1}^{\infty}\left(f, y_{k}\right) O\left(\frac{1}{k}\right)-\frac{\sqrt{2}}{2 a \pi} \frac{\left(f, y_{r}\right)}{y_{r}(1)} \sum_{k=r+1}^{\infty} \frac{(-1)^{k}}{k} \Phi_{k-1}(x)+\sum_{k=r+1}^{\infty} O\left(\frac{1}{k^{2}}\right) .
\end{gathered}
$$

It is obvious that

$$
\left|\sum_{k=r+1}^{\infty} O\left(\frac{1}{k^{2}}\right)\right| \leq \text { const } \cdot \sum_{k=r+1}^{\infty} \frac{1}{k^{2}}<+\infty .
$$

Since by Theorem 2.4 the system $\left\{y_{k}(x)\right\}_{k=1, k \neq r}^{\infty}$ is a Riesz basis in $L_{2}(0,1)$ it follows that

$$
\begin{aligned}
& \left|\sum_{k=r+1}^{\infty}\left(f, y_{k}\right) O\left(\frac{1}{k}\right)\right| \leq \text { const } \sum_{k=r+1}^{\infty}\left|\left(f, y_{k}\right)\right| \cdot \frac{1}{k} \leq \\
& \text { const }\left\{\sum_{k=r+1}^{\infty}\left|\left(f, y_{k}\right)\right|^{2}+\sum_{k=r+1}^{\infty} \frac{1}{k^{2}}\right\}^{\frac{1}{2}}<+\infty .
\end{aligned}
$$

Therefore, to study the uniform convergence of the series (5.6) it is sufficient to investigate the uniform convergence of the following series:

$$
\begin{aligned}
& \sum_{k=r+1}^{\infty}\left(f, y_{k}\right) \Phi_{k-1}(x), \\
& \sum_{k=r+1}^{\infty} \frac{(-1)^{k}}{k} \Phi_{k-1}(x) .
\end{aligned}
$$


We define the functions $Q_{1}(x), Q_{2}(x)$ and $Q_{3}(x), x \in[0,1]$, as follows:

$$
\begin{gathered}
Q_{1}(x) \equiv Q_{2}(x)=\frac{\left(q_{0}-2 / a\right) x-q_{0}(x)}{4 \pi}, \\
Q_{3}(x)=\frac{\left(q_{0}-2 / a\right) x+q_{0}(x)}{4 \pi} .
\end{gathered}
$$

Hence it follows from (5.1) that

$$
\begin{gathered}
y_{k}(x)=\Phi_{k-1}(x)+\frac{Q_{1}(x)}{k} \sin \left(k-\frac{3}{4}\right) \pi x+\frac{Q_{2}(x)}{k} \cos \left(k-\frac{3}{4}\right) \pi x- \\
\frac{Q_{3}(x)}{k} e^{-\left(k-\frac{3}{4}\right) \pi x}+O\left(\frac{1}{k^{2}}\right) .
\end{gathered}
$$

We introduce the functions $e_{k, j}(x), x \in[0,1], k \in \mathbb{N}, j=1,2,3$, which are defined by

$$
e_{k, j}(x)=\left\{\begin{array}{cc}
\sin \left(k-\frac{3}{4}\right) \pi x & \text { if } j=1, \\
\cos \left(k-\frac{3}{4}\right) \pi x & \text { if } j=2, \\
e^{-\left(k-\frac{3}{4}\right) \pi x} & \text { if } j=3 .
\end{array}\right.
$$

Then it follows from (5.13) that

$$
y_{k}(x)=\Phi_{k-1}(x)+\sum_{j=1}^{3} \frac{Q_{j}}{k} e_{k, j} .
$$

By virtue of (5.14) the series (5.11) is represented in the following form

$$
\begin{gathered}
\sum_{k=r+1}^{\infty}\left(f, y_{k}\right) \Phi_{k-1}(x)=\sum_{k=r+1}^{\infty}\left(f, \Phi_{k-1}\right) \Phi_{k-1}(x)+ \\
\sum_{j=1}^{3} \sum_{k=r+1}^{\infty} \frac{\left(f Q_{j}, e_{k, j}\right)}{k} \Phi_{k-1}(x)+\sum_{k=r+1}^{\infty} \Phi_{k-1}(x) O\left(\frac{1}{k^{2}}\right) .
\end{gathered}
$$

Note that for each $j \in\{1,2,3\}$ the system $\left\{e_{k, j}\right\}_{k=1}^{\infty}$ is a Bessel system (the Bessel property of the system $\left\{e_{k, j}\right\}_{k=1}^{\infty}$ for $j=1$ and $j=2$ is obvious, for $j=3$ follows from [26, Lemma 5]). Hence we have

$$
\left|\sum_{k=r+1}^{\infty} \frac{\left(f Q_{j}, e_{k, j}\right)}{k}\right| \leq \text { const }\left\{\sum_{k=r+1}^{\infty} \frac{1}{k^{2}}+\sum_{k=r+1}^{\infty}\left|\left(f Q_{j}, e_{k, j}\right)\right|^{2}\right\} \leq \operatorname{const}\left(1+\|f\|_{2}^{2}\right) \text {. }
$$

Thus the series (5.11) converges uniformly on [0,1], because by the hypothesis of this theorem the series $\sum_{k=r+1}^{\infty}\left(f, \Phi_{k-1}\right) \Phi_{k-1}(x)$ converges uniformly on $[0,1]$.

In view (3.13) we have

$$
\begin{gathered}
\Phi_{k}(x)=\sin \left(k+\frac{1}{4}\right) \pi x-\cos \left(k+\frac{1}{4}\right) \pi x+e^{-\left(k+\frac{1}{4}\right) \pi x}+O\left(e^{-k \pi}\right)= \\
-\sqrt{2}(-1)^{k} \cos \frac{\pi}{4}(x-1) \sin k \pi(1-x)+\sqrt{2}(-1)^{k} \sin \frac{\pi}{4}(x-1) \cos k \pi(1-x)+ \\
e^{-\left(k+\frac{1}{4}\right) \pi x}+O\left(e^{-k \pi}\right),
\end{gathered}
$$


which implies that

$$
\begin{gathered}
(-1)^{k+1} \Phi_{k}(x)=\sqrt{2} \cos \frac{\pi}{4}(x-1) \sin k \pi(1-x)- \\
\sqrt{2} \sin \frac{\pi}{4}(x-1) \cos k \pi(1-x)-(-1)^{k} e^{-\left(k+\frac{1}{4}\right) \pi x}+O\left(e^{-k \pi}\right) .
\end{gathered}
$$

It is known (see, for example, [27, p. 610]) that the functional series $\sum_{k=1}^{\infty} \frac{\sin k \pi x}{k+1}$ converges pointwise on $[0,1]$ and converges uniformly on $[b, 1]$ for any $b \in(0,1)$; the functional series $\sum_{k=1}^{\infty} \frac{\cos k \pi x}{k+1}$ converges pointwise on $(0,1]$ and converges uniformly on $[b, 1]$ for any $b \in(0,1)$. Hence the functional series $\sum_{k=1}^{\infty} \frac{\sin k \pi(1-x)}{k+1}$ and $\sum_{k=1}^{\infty} \frac{\cos k \pi(1-x)}{k+1}$ converge uniformly on $[0, c]$ for any $c \in(0,1)$.

Obviously, the numerical series $\sum_{k=1}^{\infty} \frac{(-1)^{k}}{k+1}$ converges (therefore trivially uniformly over $[0,1]$ since the $k$ th term is independent of $x)$. Also the functions $e^{-\left(k+\frac{1}{4}\right) \pi x}$ for each $x \in[0,1]$ form a monotone sequence and $0<e^{-\left(k+\frac{1}{4}\right) \pi x} \leq 1$ for any $x \in[0,1]$ and $k \in \mathbb{N}$. Therefore by the Abel's Test for Uniform Convergence the series $\sum_{k=1}^{\infty} \frac{(-1)^{k+1}}{k+1} e^{-\left(k+\frac{1}{4}\right) \pi x}$ converges uniformly on $[0,1]$.

Thus by using of equality $\Phi_{k}(1)=0, k \in \mathbb{N}$, it follows from the above consideration that the functional series

$$
\sum_{k=r+1}^{\infty} \frac{(-1)^{k} \Phi_{k-1}(x)}{k}=\sum_{k=r}^{\infty} \frac{(-1)^{k+1} \Phi_{k}(x)}{k+1}
$$

converges pointwise on $[0,1]$ and converges uniformly on $[0, c]$ for any $c \in(0,1)$.

We now prove that the uniform convergence for the series (5.17) does not hold on the interval $[0,1]$. By the above argument it suffices to consider series

$$
\sum_{k=1}^{\infty} \frac{(-1)^{k+1} \sin \left(k+\frac{1}{4}\right) \pi x-(-1)^{k+1} \cos \left(k+\frac{1}{4}\right) \pi x}{k+1}
$$

or that the same

$$
\sum_{k=1}^{\infty} \frac{\sin \left(k+\frac{1}{4}\right) \pi(1-x)}{k+1} .
$$

Now we consider the function

$$
G(u)=g(u)-\frac{u^{2}}{4}=\sin u-\frac{u^{2}}{4}, u \in \mathbb{R} .
$$

Then we have

$$
G^{\prime}(u)=\cos u-\frac{u}{2} \text { and } G^{\prime \prime}(u)=-\sin u-\frac{1}{2}, u \in \mathbb{R} .
$$

Since $G^{\prime \prime}(u)<0$ for $0 \leq u \leq \frac{\pi}{4}$ and $G^{\prime}\left(\frac{\pi}{4}\right)=\frac{\sqrt{2}}{2}-\frac{\pi}{8}>0$ it follows that the function $G(u)$ is strictly increasing on the interval $\left[0, \frac{\pi}{4}\right]$. Hence for any $u \in\left[0, \frac{\pi}{4}\right]$ 
we have $G(u) \geq G(0)=0$. Therefore, we have

$$
g(u) \geq \frac{u^{2}}{4}, 0 \leq u \leq \frac{\pi}{4}
$$

Let $x=x_{N}=\frac{8 N}{8 N+1}$, where $N$ is a natural number such that $N>r$. Then for $N \leq k \leq 2 N$ we have

$$
a_{k}=\left(k+\frac{1}{4}\right)\left(1-x_{N}\right) \pi=\left(k+\frac{1}{4}\right) \frac{\pi}{8 N+1} \leq\left(2 N+\frac{1}{4}\right) \frac{\pi}{8 N+1}=\frac{\pi}{4} .
$$

By (5.20) it follows from (5.19) that

$$
g\left(a_{k}\right) \geq \frac{a_{k}^{2}}{4} \geq \frac{(4 k+1)^{2} \pi^{2}}{64(8 N+1)^{2}} \text { for } N+1 \leq k \leq 2 N .
$$

Let $\left\{S_{k}(x)\right\}_{k=1}^{\infty}$ be the a partial sum of the series (5.18). Then by using the relation (5.21) we obtain

$$
\begin{gathered}
S_{2 N}\left(x_{N}\right)-S_{N}\left(x_{N}\right) \geq \frac{\pi^{2}}{64(8 N+1)^{2}} \sum_{k=N+1}^{2 N} \frac{(4 k+1)^{2}}{k+1} \geq \\
\frac{\pi^{2}}{8(8 N+1)^{2}} \sum_{k=N+1}^{2 N} k=\frac{\pi^{2}}{8(8 N+1)^{2}} \frac{(3 N+1) N}{2} \geq \frac{\pi^{2}}{8 \cdot 81 N^{2}} \frac{3 N^{2}}{2}=\frac{\pi^{2}}{432} .
\end{gathered}
$$

The proof of this theorem is complete.

By virtue of [21, Ch. 1, Theorem 7] and Theorem 2.4, the system $\left\{u_{k}(x)\right\}_{k=1, k \neq r}^{\infty}$ adjoint to the system $\left\{y_{k}(x)\right\}_{k=1, k \neq r}^{\infty}$ is a basis in $L_{p}, 1<p<\infty$, which is a Riesz basis for $p=2$. Then it follows that any continuous function $f(x)$ can be expanded in the following Fourier series in the system $\left\{u_{k}(x)\right\}_{k=1, k \neq r}^{\infty}$ that converges in the space $L_{p}, 1<p<\infty$ :

$$
f(x)=\sum_{k=1, k \neq r}^{\infty}\left(f, y_{k}\right) u_{k}(x)
$$

Now we study the uniform convergence of the series (5.22).

Theorem 5.2. Let $r$ be an arbitrarily fixed natural number and $f(x) \in C[0,1]$. Then the Fourier series (5.22) of function $f(x)$ in the system $\left\{u_{k}(x)\right\}_{k=1, k \neq r}^{\infty}$ is uniformly convergent on the interval $[0,1]$ if and only if the function $f(x)$ has a uniformly convergent Fourier expansions in the system $\left\{\Phi_{k}(x)\right\}_{k=1}^{\infty}$ on the interval $[0,1]$. 
Proof. By virtue of (5.2), (5.5), (5.9) and (5.15) we have

$$
\begin{gathered}
\sum_{k=r+1}^{\infty}\left(f, y_{k}\right) u_{k}(x)=\sum_{k=r+1}^{\infty}\left(f, y_{k}\right) y_{k}(x)-\frac{y_{r}(x)}{y_{r}(1)} \sum_{k=r+1}^{\infty}\left(f, y_{k}\right) y_{k}(1)+ \\
\sum_{k=r+1}^{\infty}\left(f, y_{k}\right) O\left(\frac{1}{k^{2}}\right)=\sum_{k=r+1}^{\infty}\left(f, \Phi_{k-1}\right) \Phi_{k-1}(x)+\sum_{j=1}^{3} \sum_{k=r+1}^{\infty} \frac{\left(f Q_{j}, e_{k, j}\right)}{k} \Phi_{k-1}(x)+ \\
\sum_{k=r+1}^{\infty} \Phi_{k-1}(x) O\left(\frac{1}{k^{2}}\right)-\frac{y_{r}(x)}{y_{r}(1)} \sum_{k=r+1}^{\infty}\left(f, y_{k}\right) O\left(\frac{1}{k}\right)+\sum_{k=r+1}^{\infty}\left(f, y_{k}\right) O\left(\frac{1}{k^{2}}\right) .
\end{gathered}
$$

It follows from the proof of Theorem 5.1 that the series

$$
\sum_{j=1}^{3} \sum_{k=r+1}^{\infty} \frac{\left(f Q_{j}, e_{k, j}\right)}{k} \Phi_{k-1}(x) \text { and } \sum_{k=r+1}^{\infty}\left(f, y_{k}\right) O\left(\frac{1}{k}\right)
$$

are uniformly convergent on the interval $[0,1]$ (the uniform convergence on the interval $[0,1]$ of the series $\sum_{k=r+1}^{\infty} \Phi_{k-1}(x) O\left(\frac{1}{k^{2}}\right)$ and $\sum_{k=r+1}^{\infty}\left(f, y_{k}\right) O\left(\frac{1}{k^{2}}\right)$ is obvious). Now the assertion of the theorem follows directly from (5.23). The proof of this theorem is complete.

Acknowledgements. The authors are grateful to the referee whose comments and suggestions contributed to a significant improvement in the text of the article and in the transparency of the obtained results.

\section{References}

[1] Z.S. Aliyev, Basis properties in $L_{p}$ of systems of root functions of a spectral problem with spectral parameter in a boundary condition, Differ. Equ. 47 (2011), no. 6, 766-777.

[2] Z.S. Aliyev, On basis properties of root functions of a boundary value problem containing a spectral parameter in the boundary conditions, Doklady Math. 87 (2013), no. 2, 137-139.

[3] Z.S. Aliev, A.A. Dunyamalieva, Defect basis property of a system of root functions of a Sturm-Liouville problem with spectral parameter in the boundary conditions, Differ. Equ., 51 (2015), no. 10, 1249-1266.

[4] Z.S. Aliyev, S.B. Guliyeva, Properties of natural frequencies and harmonic bending vibrations of a rod at one end of which is concentrated inertial load, J. Differential Equations 263 (2017), no. 9, 5830-5845.

[5] Z.S. Aliyev, N.B. Kerimov, V.A. Mekhrabov, On the convergence of expansions in eigenfunctions of a boundary value problem with a spectral parameter in the boundary conditions, I, Differ. Equ., 56 (2020), no. 2, 143-157.

[6] Z.S. Aliyev, N.B. Kerimov, V.A. Mekhrabov, On the convergence of expansions in eigenfunctions of a boundary value problem with a spectral parameter in the boundary conditions, II, Differ. Equ., 56 (2020), no. 3, 277-289.

[7] Z.S. Aliyev, F.M. Namazov, Spectral properties of the equation of a vibrating rod at both ends of which the masses are concentrated. Banach J. Math. Anal. 14 (2020), 585-606. 
[8] J. Ben Amara, A.A. Vladimirov, On a fourth-order problem with spectral and physical parameters in the boundary condition, Izvestiya: Mathematics, 68 (2004), no. 4, 645658.

[9] D.O. Banks, G.J. Kurowski, A Prüfer transformation for the equation of the vibrating beam, Trans. Amer. Math. Soc. 199 (1974), 203-222.

[10] D.O. Banks, G.J. Kurowski, A Prüfer transformation for the equation of a vibrating beam subject to axial forces, J. Differential Equations, 24 (1977), no. 1, 57-74.

[11] B.B. Bolotin, Vibrations in technique: Handbook in 6 volumes, The vibrations of linear systems, I, Engineering Industry, Moscow, 1978.

[12] C.T. Fulton, Two-point boundary value problems with eigenvalue parameter in the boundary conditions, Proc. Roy. Soc. Edinburgh, Sect. A, 77 (1977), no. 3-4, 293-308.

[13] D.A. Gulyaev, On the uniform convergence of spectral expansions for a spectral problem with boundary conditions of the third kind one of which contains the spectral parameter. Differ. Equ. 47 (2011), no. 10, 1503-1507.

[14] S. Janczewsky, Oscillation theorems for the differential boundary value problems of the fourth order, Ann. Math. 29 (1928), no. 2, 521-542.

[15] N.Yu. Kapustin, On a spectral problem arising in a mathematical model of torsional vibrations of a rod with pulleys at the ends, Differ. Equ., 41 (2005), no. 10, 1490-1492.

[16] N.Yu. Kapustin, On the uniform convergence of the Fourier series for a spectral problem with squared spectral parameter in a boundary condition. Differ. Equ. 46 (2010), no.10, 1504-1507.

[17] N.Yu. Kapustin, On the uniform convergence in $C^{1}$ of Fourier series for a spectral problem with squared spectral parameter in a boundary condition. Differ. Equ. 47 (2011), no. 10, 1394-1399.

[18] N.Yu. Kapustin, On the spectral problem arising in the solution of a mixed problem for the heat equation with a mixed derivative in the boundary conditions. Differ. Equ. 48 (2012), no. 5, 694-699.

[19] N.Yu. Kapustin, E.I. Moiseev, On the basis property in the space $L_{p}$ of systems of eigenfunctions corresponding to two problems with spectral parameter in the boundary condition, Differ. Equ., 36 (2000), no. 10, 1357-1360.

[20] N.Yu. Kapustin, E.I. Moiseev, A remark on the convergence problem for spectral expansions corresponding to a classical problem with spectral parameter in the boundary condition. Differ. Equ. 37 (2001), no. 2, 1677-1683.

[21] B.S. Kashin, A.A. Saakyan, Orthogonal series, Transl. Math. Monographs, Vol. 75, Amer. Math. Soc., Providence, RI 1989.

[22] N.B. Kerimov, Z. S. Aliev, Basis properties of a spectral problem with spectral parameter in the boundary condition, Sbornik: Mathematics, 197 (2006), no. 10, 14671487.

[23] N.B. Kerimov, S. Goktas, E.A. Maris, Uniform convergence of the spectral expansions in terms of root functions for a spectral problem. Elect. J. Differ. Equ. 2016 (2016), no. 80, 1-14.

[24] N.B. Kerimov, E.A. Maris, On the uniform convergence of the Fourier Series for one spectral problem with a spectral parameter in a boundary condition. Math. Methods Appl. Sci. 39 (2016), no. 9, 2298-2309.

[25] N.B. Kerimov, R.G. Poladov, Basis properties of the system of eigenfunctions in the Sturm-Liouville problem with a spectral parameter in the boundary conditions, Doklady Math., 85 (2012), no. 1, 8-13.

[26] G.M. Keselman, On the unconditional convergence of eigenfunction expansions of certain differential operators. Izv. Vyssh. Uchebn. Zaved. Mat. 39(1964), no. 2, 82-93 [in Russian]. 
[27] L.D. Kudryavtsev, Mathematical Analysis, Vol. 1, Vysshaya Shkola, Moscow, 1973 [in Russian].

[28] V.M. Kurbanov, Conditions for the absolute and uniform convergence of the biorthogonal series corresponding to a differential operator, Doklady Math. 78 (2008), no. $2,748-750$.

[29] V.M. Kurbanov, Y.I. Huseynova, On convergence of spectral expansion of absolutely continuous vector-function in eigenvector-functions of fourth order differential operator, Trans. Acad. Sci. Azerb. Ser. Phys.-Tech. Math. Sci. 34 (2014), no. 1, 83-90.

[30] E.I. Moiseev, N.Yu. Kapustin, On singularities of the root space of a spectral problem with spectral parameter in a boundary condition, Doklady Math., 385 (2002), n0. 1, 20-24.

[31] M. Möller, B. Zinsou, Self-adjoint fourth order differential operators with eigenvalue parameter dependent boundary conditions, Quaest. Math., 34 (2011), no. 3, 393-406.

[32] M.A. Naimark, Linear Differential Operators, Ungar, New York, 1967.

[33] F.M. Namazov, Uniform convergence of fourier series expansions for a fourth-order spectral problem with boundary conditions depending on the eigenparameter. Bull. Iran. Math. Soc. (2020), doi.org/10.1007/s41980-020-00378-6.

[34] E.M. Russakovskii, Operator treatment of boundary problems with spectral parameters entering via polynomials in the boundary conditions, Funct. Anal. Appl., 9 (1975), no. $4,358-359$.

[35] A.A. Shkalikov, Boundary value problems for ordinary differential equations with a parameter in the boundary conditions, J. Sov. Math., 33 (1986), 1311-1342.

[36] J. Walter, Regular eigenvalue problems with eigenvalue parameter in the boundary condition, Math. Z., 133 (1973), no. 4, 301-312.

Konul F. Abdullayeva

Sumgait State University, Sumgait AZ5008, Azerbaijan

E-mail address: konul.abdullayeva.15@mail.ru

Ziyatkhan S. Aliyev

Baku State University, Baku AZ1148, Azerbaijan

Institute of Mathematics and Mechanics NAS of Azerbaijan, Baku AZ1141, Azerbaijan

E-mail address: z_aliyev@mail.ru

Nazim B. Kerimov

Khazar University, Baku AZ1096, Azerbaijan

Institute of Mathematics and Mechanics NAS of Azerbaijan, Baku AZ1141, Azerbaijan

E-mail address: nazimkerimov@yahoo.com

Received: February 12, 2020; Revised: July 7, 2020; Accepted: September 1, 2020 